\title{
Latest Results of Double Chooz
}

\author{
Stefan Schoppmann \\ III. Physikalisches Institut, RWTH Aachen University, 52056 Aachen, Germany \\ E-mail: schoppmannephysik.rwth-aachen. de
}

The Double Chooz experiment aims for a precise measurement of the neutrino mixing angle $\theta_{13}$. It observes the disappearance of reactor antineutrinos produced at two $4.25 \mathrm{GW}_{\text {th }}$ nuclear reactors located at the CHOOZ-B power plant in Chooz, France. The experiment consists of two identical liquid scintillator detectors of $10 \mathrm{~m}^{3}$ fiducial volume. The far detector measuring the neutrino flux near its first oscillation minimum at $1050 \mathrm{~m}$ average baseline is operational since 2011 . The near detector measures the unoscillated flux at $400 \mathrm{~m}$ average baseline. It is operating since 2015 . With the new two detector setup, it is now possible to achieve a strong suppression of all systematic uncertainties to below $0.5 \%$ relative uncertainty.

In this contribution, the Double Chooz Collaboration presents the first measurement of reactor neutrino disappearance with the final two detector setup of the Double Chooz experiment. A deficit consistent with neutrino oscillations is observed in 673 days of far detector and 151 days of near detector data. The size of the neutrino mixing angle is determined to be $\sin ^{2}\left(2 \theta_{13}\right)=0.111 \pm$ 0.018 (stat + sys) yielding an exclusion of the no-oscillation scenario at 5.8 standard deviations confidence level.

XIII International Conference on Heavy Quarks and Leptons

22-27 May, 2016

Blacksburg, Virginia, USA

\footnotetext{
*Speaker.

†On behalf of the Double Chooz collaboration

‡Now at Max-Planck-Institut für Kernphysik, 69117 Heidelberg, Germany
} 


\section{Reactor Neutrino Oscillations}

15 years ago, evidence was found that neutrino oscillations, i.e. the conversion of neutrino flavours, exist $[1,2]$. Several other experiments confirmed that neutrinos change their flavour periodically as they travel. Connected with this discovery was the proof that neutrinos possess masses.

Neutrino oscillations between the three known neutrino flavour eigenstates $v_{e}, v_{\mu}$ and $v_{\tau}$ are described by 6 parameters in the simplest case. Three mixing angles $\theta_{12}, \theta_{13}$ and $\theta_{23}$ are describing the rotation between the vectors of the eigenbasis built from the flavour eigenstates and the vectors of the eigenbasis built from the mass eigenstates $v_{1}, v_{2}$ and $v_{3}$. The naming convention for the mass eigenstates is such that the order follows the magnitude of the overlap with the flavour eigenstate $v_{e}$, where $v_{1}$ shows the largest overlap with $v_{e}$ and $v_{3}$ the smallest. The three angles are connected to the magnitude of the periodical flavour conversions. Moreover, two independent neutrino mass differences $\Delta m_{31}^{2}$ and $\Delta m_{21}^{2}$ are used to describe neutrino oscillations. Here, $\Delta m_{i j}^{2}:=m_{v_{i}}^{2}-m_{v_{j}}^{2}$ denotes the difference between the squared mass of the $v_{i}$ and the $v_{j}$ neutrino mass eigenstate. Additionally, a phase $\delta_{C P}$ is describing the difference in neutrino oscillations for neutrinos and antineutrinos.

Currently, the phase $\delta_{C P}$ is unknown, i.e. it is unclear if neutrinos and antineutrinos behave differently. In addition, the sign of the mass difference $\Delta m_{31}^{2}$ is undetermined, i.e. it is unsure whether the mass eigenstate $v_{3}$ is the heaviest (normal ordering) or lightest (inverted ordering). Moreover, until 2011, no indications existed whether neutrino oscillations connected to the parameter $\theta_{13}$ are realised in nature, i.e. it was unclear whether $\theta_{13}$ was different from zero. Using a reactor-based experiment that observes the disappearance of nuclear reactor neutrinos, i.e. electron antineutrinos, it is possible to measure the parameter $\theta_{13}$ independent of the unknown phase $\delta_{C P}$. The survival probability of the initial reactor neutrinos of energy $E$ after a travel distance $L$ can be derived in good approximation as

$$
P\left(\bar{v}_{e} \rightarrow \bar{v}_{e}\right) \approx 1-\sin ^{2}\left(2 \theta_{13}\right) \sin ^{2}\left(\frac{\Delta m_{e E}^{2} L}{4 E}\right)-\cos ^{4}\left(\theta_{13}\right) \sin ^{2}\left(2 \theta_{12}\right) \sin ^{2}\left(\frac{\Delta m_{21}^{2} L}{4 E}\right)
$$

with

$$
\Delta m_{e e}^{2}:=\cos ^{2}\left(\theta_{12}\right) \Delta m_{31}^{2}+\sin ^{2}\left(\theta_{12}\right) \Delta m_{32}^{2}
$$

denoting the effective squared mass splitting observed in such an experiment. The measurement strategy of reactor-based experiments is in contrast to accelerator-based experiments that observe the $\delta_{C P}$ dependent appearance of electron neutrinos in a muon neutrino beam.

\section{The Double Chooz Experiment}

The Double Chooz experiment is a reactor neutrino disappearance experiment located at the French nuclear power plant CHOOZ-B near the French-Belgian border. It aims for the measurement of the mixing angle $\theta_{13}$. It consists of two identical liquid scintillator detectors measuring the neutrino flux from two $4.25 \mathrm{GW}_{\text {th }}$ nuclear reactors. The near detector has an approximate distance of $400 \mathrm{~m}$ to the reactor cores. It provides a first measurement of the neutrino flux. This is taken as the almost unoscillated reference flux. The far detector has a distance of approximately $1050 \mathrm{~m}$ 


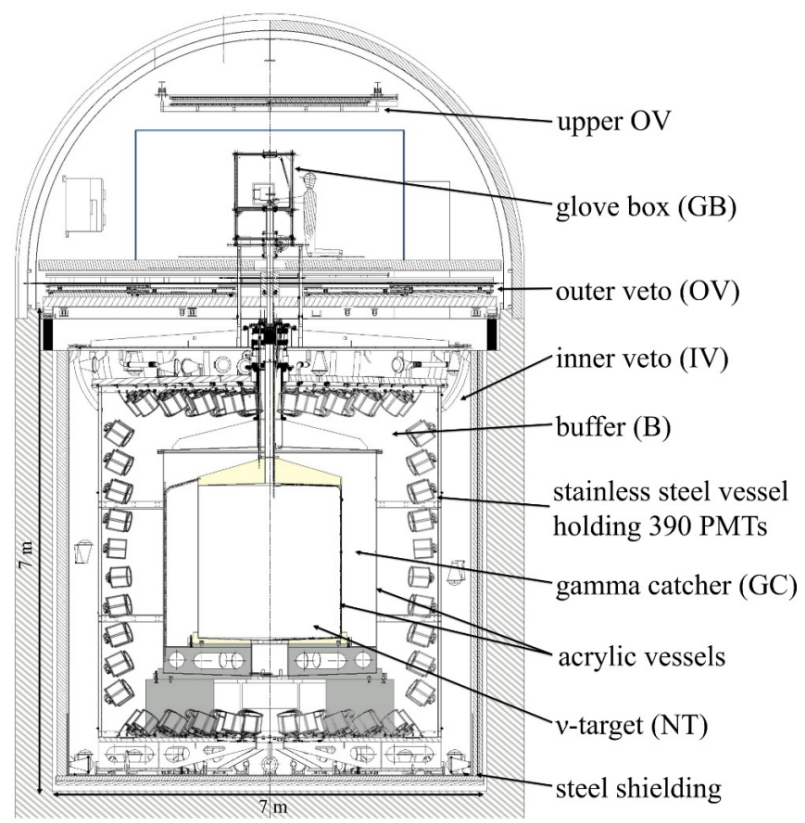

Figure 1: Sectional sketch of the Double Chooz detectors [3, p. 3].

to the cores. It is located near the first oscillation minimum such that the maximal effect of the neutrino oscillation can be measured. The far detector started operation in 2011, the near detector in 2015 .

The detectors, as depicted in Figure 1, consist of several subvolumes, each having a different purpose. The central volume is filled with $10.3 \mathrm{~m}^{3}$ liquid scintillator doped with Gadolinium. It is called neutrino target and it is the fiducial volume for the main analysis, as described later. It is accommodated in a cylindrical vessel built from optically transparent acrylics. The surrounding volume, called gamma catcher, is enclosed by another acrylic cylinder and filled with $22.4 \mathrm{~m}^{3}$ undoped liquid scintillator. It becomes relevant whenever a neutrino interacts close to the boundary of the fiducial volume. In this case, it allows to convert gamma photons into visible light even when they escape from the fiducial volume. The gamma catcher is also used as an extension of the fiducial volume in the so called Hydrogen analysis, which is described in reference [5]. The gamma catcher is enclosed by a hollow cylinder built from steel and filled with $100 \mathrm{~m}^{3}$ non-scintillating mineral oil, the buffer volume. In this volume, 390 PMTs of 10 inch diameter are installed, each oriented towards the centre of the fiducial volume. The non-scintillating mineral oil ensures that radioactive events happening in the vicinity of the PMTs, e.g. in the PMT glass or the steel vessel, are not producing light.

Optically separated from those three inner detector volumes is the inner veto volume. It is of hollow cylindrical shape equipped with 78 PMTs of 8 inch diameter and is filled with $90 \mathrm{~m}^{3}$ undoped liquid scintillator. It is a multi-purpose veto and is able to detect a variety of particles penetrating the detector from almost all directions. Around the inner veto, a passive shielding is installed. While the top shielding is made from steel in both detectors, the lateral and bottom shielding is made from different materials in the two detectors. In the far detector, steel is used 
as for the top shielding. In the near detector a water shielding is installed. Above the described cylindrical part of the detector, a planar detector built from plastic scintillator strips is established. This outer veto detector is able to identify atmospheric muons entering the cylindrical detector and its surrounding rock from the top. It consists of two layers, one being place directly above the top shielding and one being installed approximately 3 metres above.

In the Gadolinium-doped scintillator, i.e. the fiducial volume, neutrinos are detected by the inverse $\beta$-decay reaction:

$$
\bar{v}_{\mathrm{e}}+\mathrm{p}^{+} \rightarrow \mathrm{e}^{+}+\mathrm{n}
$$

This reaction can take place if the neutrino has a kinetic energy above $1.8 \mathrm{MeV}$. Below this threshold there is not enough energy present to generate the masses of the positron and the neutron. The produced positron annihilates promptly with an electron of the liquid scintillator yielding a first light entry in the detector. The produced neutron gets eventually captured by a Gadolinium nucleus yielding a second light entry. The light from the neutron capture is delayed with respect to the light of the annihilation because the capture process only starts to happen after $10 \mu \mathrm{s}$ when the neutron has thermalised. The capture process itself happens with a time constant of about $30 \mu \mathrm{s}$. The delayed light deposition yields an energy peaking around $8 \mathrm{MeV}$ while the prompt energy ranges between $1 \mathrm{MeV}$ to $11 \mathrm{MeV}$. This wide range is owed to the transfer of kinetic energy from the neutrino, which is mainly propagated to the positron. Apart from the capture by Gadolinium, the neutron can also be captured by Hydrogen present in the liquid scintillator. This yields a lower capture energy after a longer average capture time. Those captures are rejected in the main analysis presented in this contribution.

A signal similar to the inverse $\beta$-decay can be produced by a variety of backgrounds. The sources of those backgrounds are either atmospheric muons or intrinsic radioactivity. Atmospheric muons are able to fissure atoms in the surrounding rock. As consequence, fast neutrons are produced. Those can enter the detector and perform a proton recoil. The recoil mimics the prompt event while the subsequent capture of the neutron mimics the delayed event. The fast neutrons can also enter in a burst such that the prompt event is given by an additional neutron capture. As product of the fission, also light nuclei can be formed. Among those Lithium- 9 and Helium- 8 are most relevant. These two nuclei perform a $\beta$-decay followed close in time by a subsequent neutron emission of the respective daughter nucleus. The emitted electron can be identified as the prompt event and the neutron as the delayed event. In addition to the aforementioned backgrounds, the muons themselves can introduce a background when they stop and decay in the detector. The end of their trajectory gives the prompt event while the produced Michel-electron gives the delayed event. Complementing the described correlated background types, an accidental coincidence can always happen between an energy deposition by a radioactive decay and a capture of a spallation neutron. Most backgrounds are vetoed by their energy depositions in the inner or outer veto systems or they are discriminated based on high level information as will be explained in the next section.

In order to measure the disappearance of reactor neutrinos, i.e. the oscillation of neutrinos, the Double Chooz experiment uses spectral shape and rate information. In both detectors, the spectral shape and rate of the measured inverse $\beta$-decay candidates is compared to the expected rate and shape of signal and background events in 40 energy bins. The background expectation is derived 
from in situ measurements of all backgrounds. The signal expectation is based on minute-wise measurements of the thermal power of the two reactor cores and includes detailed knowledge of the fuel composition of each reactor and the fuel burn-up. Additionally, the measurement of the Bugey 4 experiment [6] is used as external information to normalise the neutrino flux prediction.

\section{New Result for $\theta_{13}$}

The commissioning of the near detector marks the begin of a new era in precision for the Double Chooz experiment. The measurement of the almost unoscillated neutrino flux in $400 \mathrm{~m}$ distance reduces the neutrino flux uncertainty significantly, as will be shown later.

After a pre-cleaning of the data, signal events are selected by four main cuts aiming for the characteristics of the inverse $\beta$-decay reaction. The inverse $\beta$-decay is characterised by a prompt energy deposition between $1 \mathrm{MeV}$ and approximately $11 \mathrm{MeV}$ followed by a delayed energy deposition of on average $8 \mathrm{MeV}$ for neutron captures by Gadolinium. Thus, a cut on prompt energy of $0.5 \mathrm{MeV}<\mathrm{E}_{\mathrm{vis}}^{\text {prompt }}<20 \mathrm{MeV}$ and on delayed energy of $4.0 \mathrm{MeV}<\mathrm{E}_{\mathrm{vis}}^{\text {delayed }}<10.0 \mathrm{MeV}$ is utilised. The large cut window on the prompt energy $E_{v i s}^{\text {prompt }}$ is chosen in order to provide a sufficient amount of background dominated analysis bins. This is found to yield a smaller uncertainty as background rates can be constraint better in the final analysis step: The energy region around $9 \mathrm{MeV}$ is dominated by Lithium-9/Helium- 8 background and the high energy bins are dominated by fast neutrons/stopping muons. Both regions thus allow to constrain the two backgrounds separately. The lower energy bound of $0.5 \mathrm{MeV}$ was determined to be large enough for showing negligible trigger inefficiency. The delayed energy signal cut $E_{v i s}^{\text {delayed }}$ was optimised for minimal contamination by fast neutron background in the high energy part and high signal efficiency in the low energy part.

The neutron capture happens after a thermalisation period of around $10 \mu \mathrm{s}$, with a time constant of $30 \mu \mathrm{s}$ for neutron captures by Gadolinium. Therefore, a delay time cut of $0.5 \mu \mathrm{s}<\Delta \mathrm{T}_{\text {delay }}<$ $150.0 \mu \mathrm{s}$ is imposed. The time delay cut $\Delta T_{\text {delay }}$ was optimised at short times to suppress stopping muon events on the one hand, while respecting the increased capture probability of neutrons due to the end of their thermalisation process on the other hand. An additional spatial distance cut between prompt and delayed event of $\Delta R_{\text {delay }}<100 \mathrm{~cm}$ is introduced to reject accidental background

\begin{tabular}{|c|c|c|c|c|}
\hline & FD-I & FD-I reactor off & FD-II & ND \\
\hline predicted IBD signal / $\mathrm{d}^{-1}$ & $38.04 \pm 0.67$ & $0.217 \pm 0.065$ & $40.39 \pm 0.69$ & $280.5 \pm 4.7$ \\
\hline accidental bkgrd / d ${ }^{-1}$ & \multicolumn{2}{|c|}{$0.070 \pm 0.003$} & $0.106 \pm 0.002$ & $0.344 \pm 0.002$ \\
\hline fast-n+stop- $\mu$ bkgrd / d ${ }^{-1}$ & \multicolumn{3}{|c|}{$0.586 \pm 0.061$} & $3.42 \pm 0.23$ \\
\hline${ }^{9} \mathrm{Li}+{ }^{8} \mathrm{He}$ bkgrd $/ \mathrm{d}^{-1}$ & \multicolumn{3}{|c|}{$0.97_{-0.16}^{+0.41}$} & $5.01 \pm 1.43$ \\
\hline total prediction / $\mathrm{d}^{-1}$ & $39.63 \pm 0.73$ & $1.85 \pm 0.30$ & $42.06 \pm 0.75$ & $289.3 \pm 4.9$ \\
\hline measured IBD cand. / $\mathrm{d}^{-1}$ & 37.64 & 0.97 & 40.29 & 293.4 \\
\hline measured IBD cand. & 17351 & 7 & 8551 & 44233 \\
\hline lifetime / d & 460.93 & 7.24 & 212.21 & 150.76 \\
\hline
\end{tabular}

Table 1: Inverse $\beta$-decay (IBD) candidate sample and prediction for signal and backgrounds. 
coincidences that are likely to happen at much larger distances between prompt and delayed vertex than proper inverse $\beta$-decay events.

Complementing the cuts presented above, several cuts are installed to further discriminate backgrounds. The main strategy is to reject high energy events and events following within $1 \mu \mathrm{s}$ after those events. By this, muon events and events induced by muons are rejected. In addition, homogeneity in the PMT hit times and charge distributions are evaluated to reduce backgrounds due to artificial light produced by the PMTs themselves. Moreover, event multiplicity, vertex position and other variables are evaluated in independent or dependent ways to reject special kinds of background events.

After the event selection, four data samples with individually estimated signal and background contributions exist. These are the dataset collected with the far detector during the single detector phase (FD-I), the two datasets collected with the far and near detector, respectively, during the two detector phase (FD-II and ND, respectively) and an additional dataset collected during a phase of inactive reactors (FD-I reactor off). The number of inverse $\beta$-decay candidates, signal and background predictions and lifetimes of the datasets can be seen in Table 1. The energy spectra of the datasets are depicted in Figure 2.
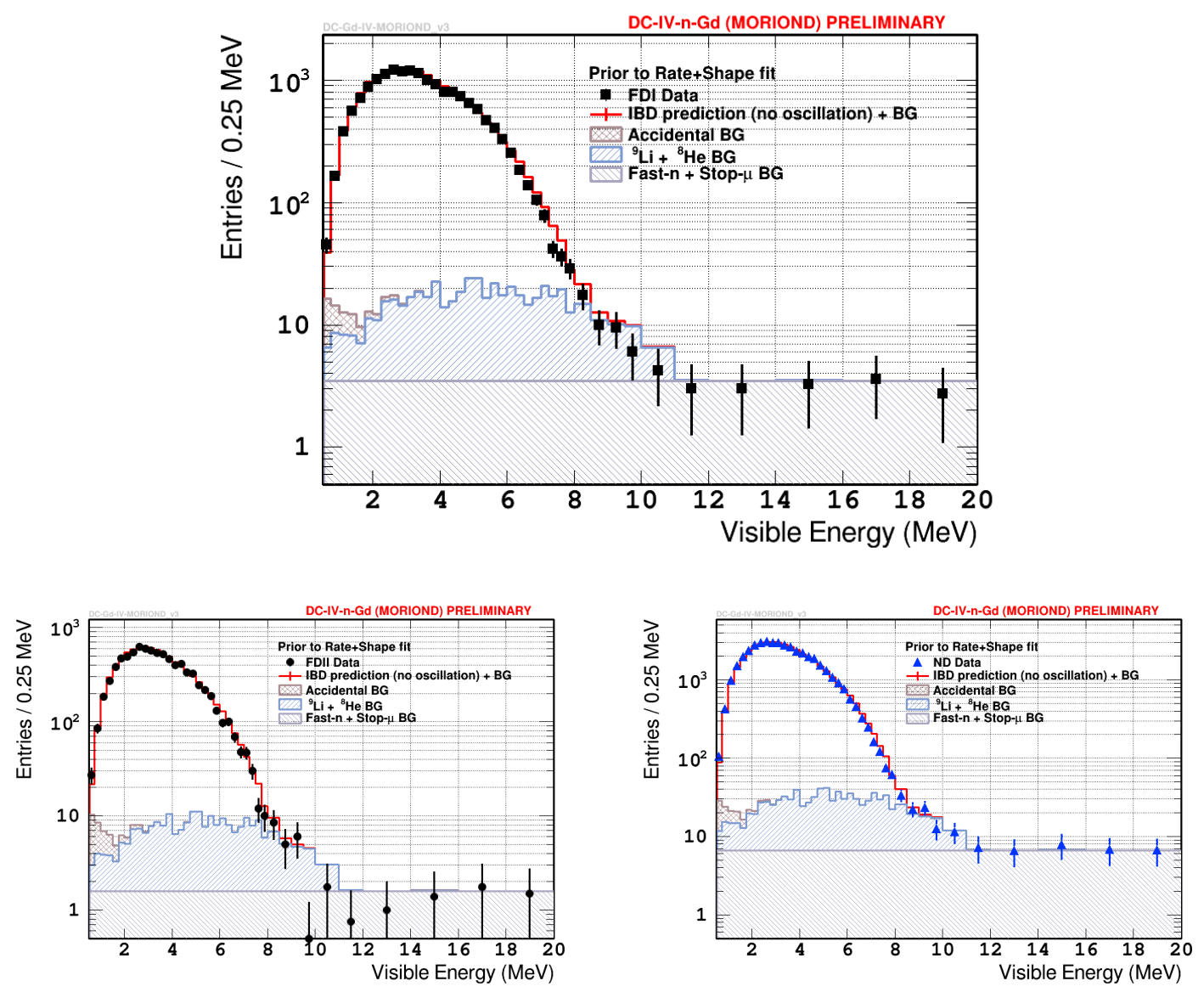

Figure 2: Input spectra for all datasets. All spectra are shown prior to the oscillation analysis using the nominal background models and assuming no neutrino oscillations. 

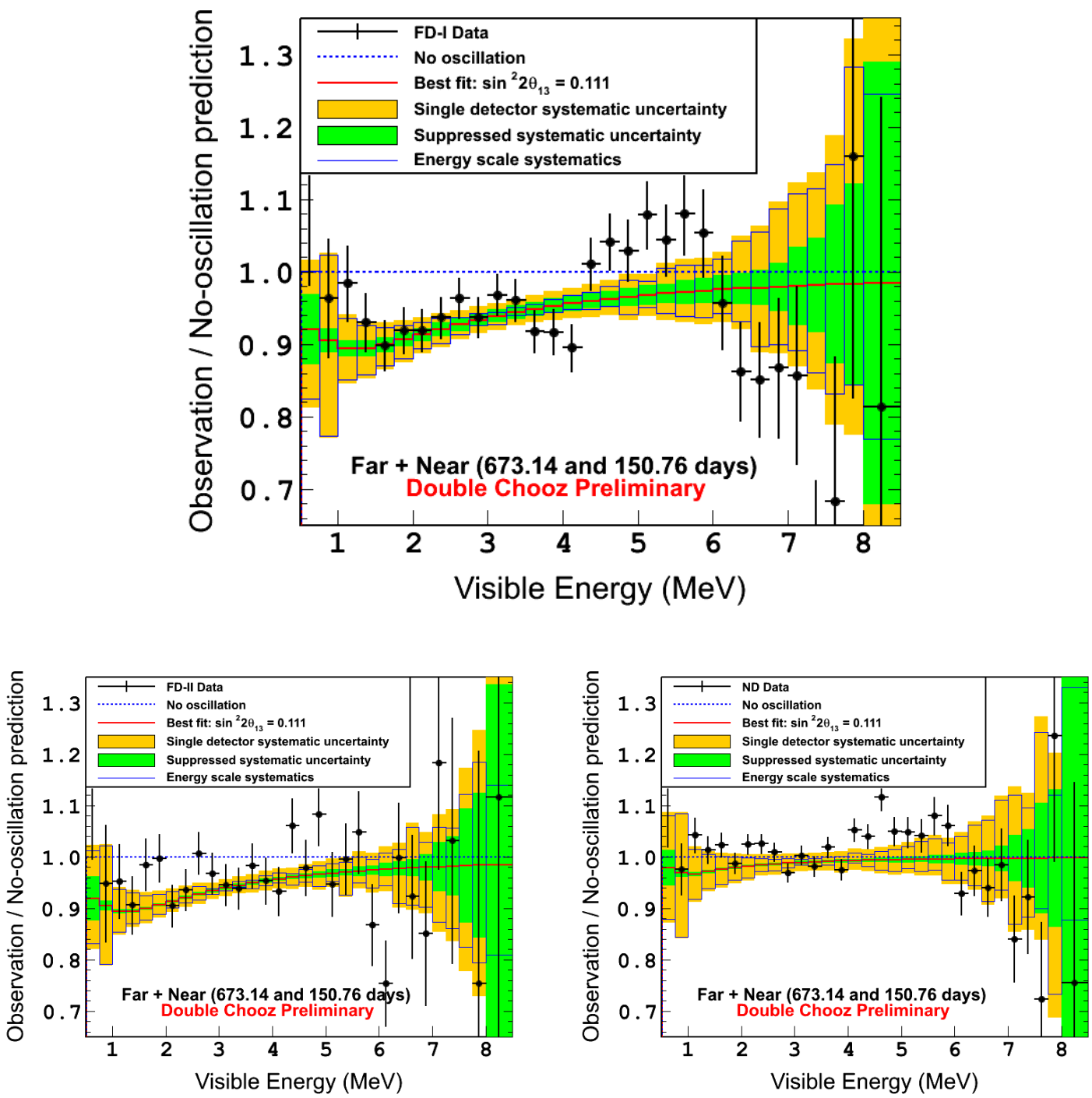

Figure 3: Best-fit spectra for all datasets. The depicted best-fit model does not include corrections in the reactor flux.

While the signal-dominated FD-I, FD-II and ND datasets are used for the determination of $\theta_{13}$, the background-dominated reactor off dataset is used to constrain the backgrounds in the oscillation analysis alongside the dedicated high energy regions of the signal-dominated datasets. At the current time, the collected and analysed data during the two detector phase extends the dataset of the single detector phase (previously published in [3]) by 9 months of runtime. Due to less overburden and consequently higher background rates and veto times in the near detector, the lifetimes of the FD-II and ND datasets differ significantly as shown in Table 1.

Due to strong correlations between the far and near detector, the new two detector setup allows to suppress the previously dominant uncertainty of the reactor flux prediction from $1.7 \%$ to $0.1 \%$. This suppression is achieved mainly due to two properties: The simultaneous run times of near 
and far detector and their almost iso-flux condition, i.e. the observation of almost the same ratio of neutrino flux from the two nuclear reactors. Both properties ensure that reactor-wise uncorrelated contributions have similar impact on both detectors. Likewise, the identical design of the two detectors allows to suppress uncertainty of the detection efficiency from $0.5 \%$ to $0.3 \%$. Here, mainly the impact of geometrical effects is reduced. In future analyses, using the fact that the liquid scintillator is identical in all detectors will further reduce uncertainties. However, this is not yet included in the current analysis stage. When performing the oscillation analysis, the background uncertainty can also be reduced from initially $0.8 \%$ to $0.4 \%$ by including the reactor-off dataset and the dedicated energy regions as mentioned above.

In the oscillation analysis, the measured number of inverse $\beta$-decay candidates in all datasets is compared simultaneously to the expected number of those events. All background expectations are derived from in situ measurements. The signal expectations are derived from reactor simulations using the thermal power of each reactor as well as detector simulations. With exception of the Lithium-9/Helium-8 event rate, all expectations are also used as constraints in the oscillation analysis. Moreover, the squared difference in neutrino masses is constrained by external data of the MINOS collaboration [7]. The oscillation analysis yields that the observed deficit of inverse $\beta$-decay candidates is consistent with a value of the mixing angle $\sin ^{2}\left(2 \theta_{13}\right)=0.111 \pm 0.018$ (stat.+syst.) using a constraint of $\Delta m_{e e}^{2}=(2.44 \pm 0.09) 10^{-3} \mathrm{eV}^{2}$. The corresponding best-fit model as depicted in Figure 3 shows a goodness of fit of $\frac{\chi^{2}}{d o f}=\frac{128.8}{120}$. The analysed data disfavours the no-oscillation hypothesis at a confidence level of 5.8 standard deviations, thereby confirming the discovery of reactor neutrino oscillations.

In order to cross-check the result, independent approaches have been developed using different implementations of systematic and statistical uncertainties. The approaches give consistent results. Moreover, for each dataset the energy scale correction, which is optimised during the oscillation analysis, has been treated uncorrelated across all datasets as validation that the two detectors have been built identically. The results for all energy scale corrections are consistent across all detectors, confirming that the two detectors have been built identically.

\section{Acknowledgments}

We thank the French electricity company EDF; the European fund FEDER; the Région de Champagne de Champagne Ardenne; the Département des Ardennes; and the Communauté de Communes Ardenne Rives de Meuse. We acknowledge the support of the CEA, CNRS/IN2P3, the computer centre CCIN2P3, and LabEx UnivEarthS in France (ANR-11-IDEX-0005-02); the Ministry of Education, Culture, Sports, Science and Technology of Japan (MEXT) and the Japan Society for the Promotion of Science (JSPS); the Department of Energy and the National Science Foundation of the United States; U.S. Department of Energy Award DE-NA0000979 through the Nuclear Science and Security Consortium; the Ministerio de Economía y Competitividad (MINECO) of Spain; the Max Planck Gesellschaft, and the Deutsche Forschungsgemeinschaft DFG, the Transregional Collaborative Research Center TR27, the excellence cluster "Origin and Structure of the Universe", and the Maier-Leibnitz-Laboratorium Garching in Germany; the Russian Academy of Science, the Kurchatov Institute and RFBR (the Russian Foundation for Basic Research); the Brazilian Ministry of Science, Technology and Innovation (MCTI), the Finan- 
ciadora de Estudos e Projetos (FINEP), the Conselho Nacional de Desenvolvimento Científico e Tecnológico (CNPq), the São Paulo Research Foundation (FAPESP), the Minas Gerais State Research Foundation (FAPEMIG, project CEX-APQ-01439-14), and the Brazilian Network for High Energy Physics (RENAFAE) in Brazil.

\section{References}

[1] Y. Ashie et al. (Super-Kamiokande Collaboration), Measurement of atmospheric neutrino oscillation parameters by Super-Kamiokande I Physical Review D 71 (2005) 11 [hep-ex/ 0501064 ]

[2] Q. R. Ahmad et al. (SNO Collaboration), Direct Evidence for Neutrino Flavor Transformation from Neutral-Current Interactions in the Sudbury Neutrino Observatory, Physical Review Letters 89 (2002) 1 [nucl-ex/0204008]

[3] Y. Abe et al. (Double Chooz Collaboration), Improved measurements of the neutrino mixing angle $\theta_{13}$ with the Double Chooz detector, Journal of High Energy Physics 10 (2014) 086, Erratum-ibid. [4] [hep-ex/1406.7763]

[4] Y. Abe et al. (Double Chooz Collaboration), Erratum to: Improved measurements of the neutrino mixing angle $\theta_{13}$ with the Double Chooz detector, Journal of High Energy Physics 02 (2015) 074 [hep-ex/1406.7763]

[5] Y. Abe et al. (Double Chooz Collaboration), Measurement of $\theta_{13}$ in Double Chooz using neutron captures on hydrogen with novel background rejection techniques, Journal of High Energy Physics 01 (2016) [hep-ex/1510.08937]

[6] Y. Declais et al., Study of reactor anti-neutrino interaction with proton at Bugey nuclear power plant, Physics Letters B 338 (1994) 2-3

[7] P. Adamson et al. (MINOS Collaboration), Combined analysis of $v_{\mu}$ disappearance and $v_{\mu} \rightarrow v_{e}$ appearance in MINOS using accelerator and atmospheric neutrinos, Physical Review Letters 112 (2014) [hep-ex/1403.0867] 